\title{
"My Invisalign experience": content, metrics and comment sentiment analysis of the most popular patient testimonials on YouTube
}

\author{
Christos Livas $^{1 *}$, Konstantina Delli ${ }^{2}$ and Nikolaos Pandis 3,4
}

\begin{abstract}
Background: The aim of the study was to investigate the popularity, content of Invisalign patient testimonials on YouTube, as well as the sentiment of the related comments.

Methods: Using the term "Invisalign experience," the top 100 results on YouTube by view count were screened for English spoken patient videos that attracted comments. Video information (time since video upload, sponsorship), engagement metrics (comments, likes, dislikes, subscriptions), and views were collected. Videos were rated for information completeness (ICS), and comments were classified by origin and content. The emotional loading of the comments was measured using automated sentiment analysis.

Results: The 40 reviewed testimonials scored an average ICS of 3.78 (SD 0.97). ICS, time since upload, and video duration did not appear to significantly influence the number of views, subscriptions, likes, dislikes, and comments. There was a statistically significant difference $(P=0.03)$ between mean positive $(2.01, \mathrm{SD} 0.95)$ and negative sentiment scores (- 1.90, SD 1.14). Commenter's status and overall comment on video were significantly associated with positive sentiment scores. There was a significant association between sponsorship, commenter's status, overall comment on video, focus of concern, perceived Invisalign's disadvantages, and increased negative sentiment scores.

Conclusions: Engagement of audience and views of the most popular Invisalign patient testimonials were not significantly influenced by completeness of information, video duration, and lifespan. The sentiment of viewers' comments about Invisalign treatment was significantly more positive and was significantly associated with their status, content, and sponsorship of videos. Orthodontic trends on YouTube need to be cautiously monitored for planning interventions that improve patients' knowledge about orthodontics.
\end{abstract}

\section{Background}

Personal stories of patients in the form of narratives, testimonials, or anecdotes are increasingly becoming available to the public as YouTube videos and discussion posts on social media and peer-support groups. Such anecdotal information is heavily weighed by health consumers when making treatment choices [1]. Health video blogs in video hosting services, also called vlogs, have the potential to impact patients' psychological

\footnotetext{
* Correspondence: c.livas@acta.nl

'Department of Orthodontics, Academic Centre for Dentistry Amsterdam (ACTA), University of Amsterdam and VU University Amsterdam, Amsterdam, The Netherlands

Full list of author information is available at the end of the article
}

health, promote health education for youth and young adults, and improve health information literacy [2].

With over a billion users, YouTube is the fastest growing video sharing Web platform, ranked second in Internet traffic worldwide and in the United States (US) [3]. The educational value of YouTube in dentistry has been reported as underdeveloped and underestimated [4]. The quality and completeness of the dental information on YouTube have been disputed due to the minimal filtering of the uploaded material [5]. Numerous orthodontic videos are also available on YouTube, mostly originating from patients [4], and reflecting a general proorthodontics attitude. Referring orthodontic patients to YouTube for relevant audiovisual information resulted in 
a significant improvement in patient knowledge compared to verbal and written instructions [6].

Given YouTube is being largely used as a primary source of orthodontic information [4], it can be presumed that laypersons interested in contemporary systems like Invisalign are likely to access the videosharing platform to answer their queries. Nowadays, $89 \%$ of US practices perform treatment with clear aligners, while the number of cases has almost doubled within a few years [7]. This upward trend did not slow down regardless of the limited evidence on the effectiveness of Invisalign compared to conventional orthodontics [8]. Interestingly, the manufacturer has recently announced to have reached 4.5 million Invisalign patients worldwide [9]. Without doubt, the growth rate of Invisalign suggests its continued popularity among clinicians and patients. It can be speculated that new communication technologies like YouTube and other social media might have contributed to the broad acceptance by patients, and especially at younger ages.

So far, the available Invisalign information on YouTube videos uploaded by patients-vloggers and the interaction between vloggers and viewers have not been investigated. Therefore, the aims of this study were (i) to determine the completeness of information of the most viewed YouTube patient testimonials regarding Invisalign treatment and the emotional content of viewers' comments and (ii) to evaluate the association patterns between video metrics, information completeness, and sentiment of comments.

\section{Methods}

\section{Search strategy}

YouTube (http://www.youtube.com) was searched on June 10, 2017, using the phrase "Invisalign experience." Preliminary YouTube search using the specific search phrase delivered more relevant results than "Invisalign" and "Invisalign testimonials" and therefore selected for the study. Before searching, the computer history and cookies were deleted. No filters regarding video upload date, type, duration, and features were enabled. The initial search returned 61,300 results. To reproduce a standard YouTube search of the average user $[10,11]$, the first 100 videos sorted by view count were screened for relevance independently by two researchers and any disagreement was resolved in a consensus meeting. The following exclusion criteria were applied: language other than English, videos without comments, no personal experience narrative, irrelevant to Invisalign information, testimonials produced by non-patients, insulting wording, poor audiovisual quality, and duplicates.

\section{Data collection}

Video information (title, hyperlink, vlogger's name, time since upload), engagement metrics (comments, likes, dislikes, subscriptions), and view metrics were recorded for all eligible videos. The description field of each video was scrutinized for sponsorship statements or endorsement deals.

All videos were viewed and available information regarding clinician's status, Invisalign cost, treatment procedure, complications and comparison between Invisalign and fixed appliances was extracted. Such information has been previously found to attract attention on social media [12]. Subsequently, two researchers (C.L. and K.D.) measured independently the information completeness score (ICS) of the videos, namely rated the videos with 1 point for each of the five abovementioned topics covered by the vlogger. Commenter's status was classified according to the categories displayed in Table 1. To prevent misinterpretation of the results regarding the target of the comments, only the ones including the word "Invisalign" were retrieved [13]. Comments were further classified by overall comment, focus of concern, and Invisalign's disadvantages as perceived by the viewer (Table 2).

\section{Sentiment analysis}

Sentiment analysis or opinion mining is an approach that classifies comments as praise remarks or complaints and can further process these classifications into actionable areas to improve clinical practice [14]. Sentiment analysis of viewers' comments on Invisalign was carried out using SentiStrength software (version 2.2, copyright Professor M. Thelwall, Faculty of Science and Engineering, University of Wolverhampton, UK), a sentiment analysis tool widely applied to sentiment detection on social network sites [15-19]. SentiStrength [20] is a lexicon-based classifier that synthesizes additional (non-lexical) linguistic information and rules to measure the strength of positive

Table 1 Commenter's status according to personal experience with or interest in Invisalign

Viewer status

Invisalign patient (former or present)

Fixed appliance patient (former or present)

Experienced both types of appliances

Interested in orthodontics

Getting Invisalign soon

Getting fixed appliances soon

Eager to get Invisalign

Willing to get but cannot afford Invisalign

Regretted getting Invisalign

Other

Not specified 
Table 2 Comment classification

\begin{tabular}{lll}
\hline Overall comment & Focus of concern & Invisalign's disadvantages \\
\hline $\begin{array}{l}\text { Found video useful/informative } \\
\text { Commented on vlogger's reliability/ }\end{array}$ & Treatment cost & Pain \\
$\begin{array}{l}\text { Positive comment on vlogger's } \\
\text { treatment outcome }\end{array}$ & Complications (pain, lisp, etc.) & Bad odor \\
$\begin{array}{l}\text { Negative comment on vlogger's } \\
\text { treatment outcome }\end{array}$ & Treatment duration/wearing time & Wearing time/commitment \\
$\begin{array}{l}\text { Confused Invisalign with retainers } \\
\text { Asked for further information }\end{array}$ & Cleaning aligners/oral hygiene & Dietary consequences \\
Other & Retention/stability & Public embarrassment \\
Combination & Efficiency & Treatment cost \\
Treatment procedures & Oral symptoms \\
No comment & If Invisalign indicated for own & Not indicated for all cases \\
& malocclusion/asked for advice & Other (e.g., enamel decalcification) \\
& Asked/shared information about own & Combination \\
& Reason for choosing Invisalign & No disadvantages mentioned
\end{tabular}

and negative sentiment in short informal English texts on a scale of \pm 1 (neutral) to \pm 5 (extremely positive/extremely negative). For instance, a text scored with $3,-5$ would signify moderate positive and very strong negative emotions. The full text of comments was imported into the Sentistrength tool, which automatically generated binary reports of positive and negative sentiment strengths.

\section{Statistical analysis}

Paired $t$ test was used to compare the means of positive and negative sentiment scores. Median regression analysis was used to investigate the association between ICS and engagement and view metrics. To determine the association between positive and negative sentiment scores and sponsorship, viewer's status, overall comment, focus of concern, and disadvantages as perceived by viewers, Pearson's chi-squared test was used. The significance level was set at 5\%. Statistical analysis was performed using a statistical software package (STATA 14.2, Stata Corporation, College Station, Tex, USA).

\section{Results}

YouTube search initially yielded 61,300 results. After screening video titles and watch pages of the first 100 videos, the list of results reduced by 19 videos (Fig. 1). After watching the remainder videos and screening the comments posted by viewers, 40 videos were considered eligible for the study.

\section{Engagement, view metrics, and sponsorship}

The 40 videos were created by 34 unique vloggers with a median of 2605.5 subscriptions. Descriptive statistics (median, interquartile range; IQR) of video duration, lifespan, and engagement metrics are displayed in Table 3. In total, 663 comments were considered for the purposes of the study. Align Technology Inc. was acknowledged in one video, whereas one vlogger revealed to have received discount for treatment costs. Five vloggers disclosed information about the Invisalign provider (name, practice, or Web site). Lack of sponsorship was clearly stated in nine testimonials.

Presentation, completeness of information, and video scores The analyzed videos presented a mean ICS of 3.78 (SD 0.97). Eleven videos achieved the theoretical maximum score of 5 points. The results of median regression analysis showed no significant association between ICS, video duration, and time since upload and either engagement metrics or views (Table 4). There was a weak evidence of significant association between video duration and view counts $(\beta$-coefficient $=0.16, P=0.10)$.

Half of the vloggers were treated by orthodontists and 17 by dentists. Twenty-nine vloggers were in active treatment at the time the video was recorded. Most vloggers discussed treatment aspects like frequency of changing trays/practice visits, attachments, wearing time, and consultation. Procedures like ClinCheck and Interproximal Enamel Reduction (IER) were scarcely reported. Pain, tooth sensitivity, or discomfort were the most frequent side-effects reported by 22 vloggers. Eleven vloggers complained about oral symptoms, and specifically, mouth dryness and soft tissue irritation. Eleven vloggers had to adjust their dietary habits, while 


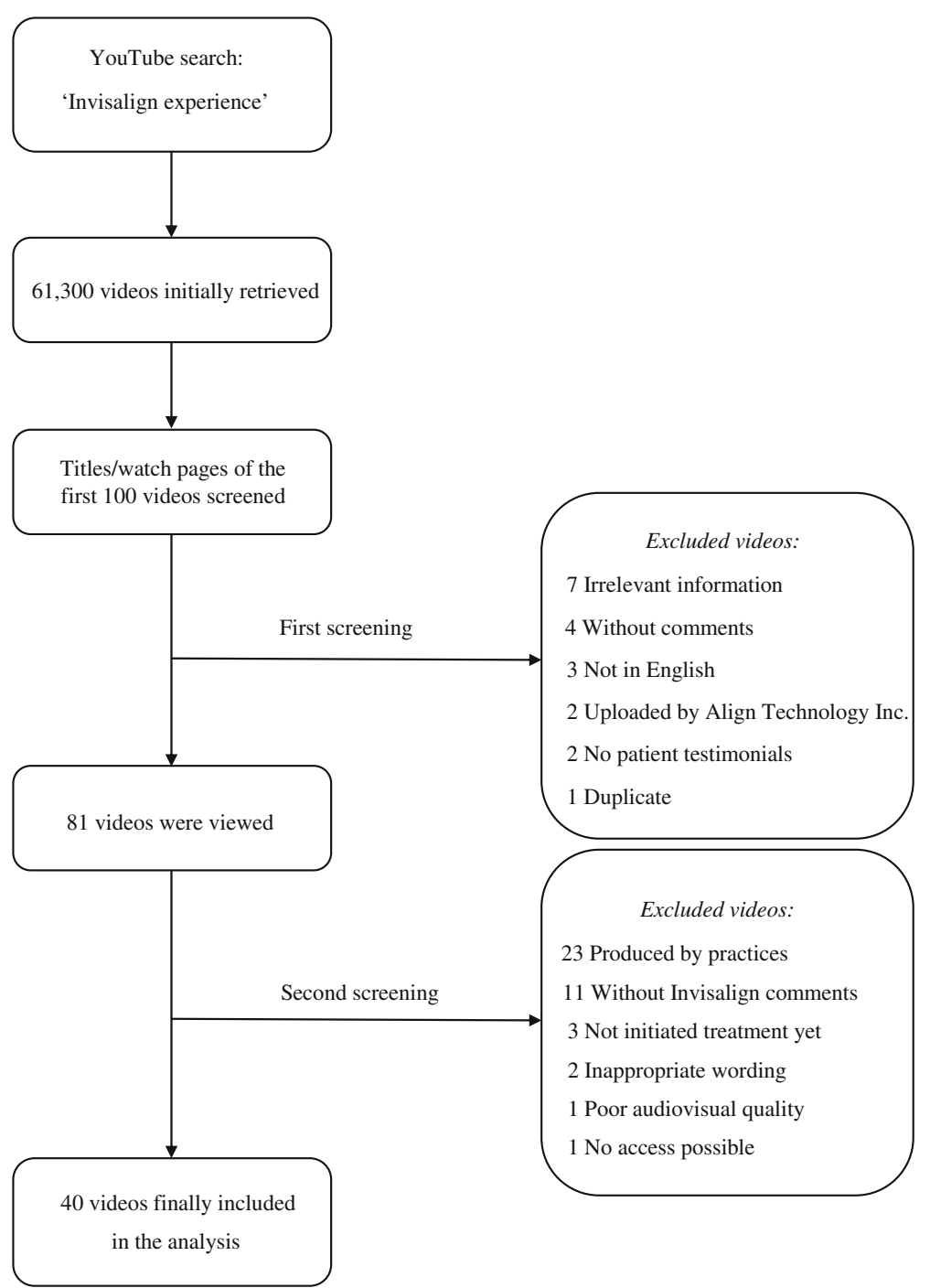

Fig. 1 Flowchart diagram of the selection process

Table 3 Descriptive statistics (median, interquartile range; IQR) of video duration, time since upload, and engagement metrics

\begin{tabular}{lll}
\hline & Median & IQR \\
\hline Duration (sec) & 526.0 & 417.5 \\
Time since upload (days) & 720.0 & 937.5 \\
Views & $10,940.0$ & $46,529.0$ \\
Subscribe & 2605.5 & 7593.5 \\
Likes & 94.0 & 160.5 \\
Dislikes & 5.0 & 25.5 \\
Comments & 33.0 & 56.5 \\
\hline
\end{tabular}

Table $4 P$ values from the median regression analysis for information completeness score (ICS), views, duration (in seconds), and time since upload (in days)

\begin{tabular}{llllll}
\hline ICS & \multicolumn{5}{l}{$P$ value } \\
\cline { 2 - 6 } & Views & Subscribe & Likes & Dislikes & Comments \\
\hline 3 points & 0.99 & 1.00 & 0.97 & 0.96 & 0.98 \\
4 points & 0.98 & 1.00 & 0.99 & 0.99 & 0.98 \\
5 points & 0.90 & 1.00 & 0.90 & 0.79 & 0.87 \\
Duration & 0.85 & 0.94 & 0.10 & 0.51 & 0.21 \\
Time since upload & 0.26 & 0.87 & 0.63 & 0.52 & 0.67 \\
\hline
\end{tabular}


speech was affected (lisp) in ten cases. Fifty percent of the vloggers compared Invisalign and fixed appliances, whereas most of them (14 out of 20) did not have personal experience with braces.

\section{Comment content and sentiment analysis}

Commenter's status was not specified in 234 cases, while many commenters were related to Invisalign treatment (i.e., 163 former or present patients, 67 motivated to get Invisalign, 41 planning to start treatment soon).

Four hundred sixty-nine viewers did not comment on the video itself, 80 found the video useful/informative, and 31 commented on vlogger's reliability/sponsoring. Most commenters focused on asking or sharing information about own malocclusion/treatment or even finding similarities with the vlogger's condition, treatment cost, complications, and efficiency. Twenty-four percent of the viewers shared their view on Invisalign's disadvantages with most of them mentioning cost, a combination of disadvantages, and pain/oral symptoms.

There was a statistically significant difference $(P=0.03)$ between mean positive (2.01, SD: 0.95$)$ and negative sentiment scores $(-1.90$, SD 1.14). There was a significant association between commenter's status, overall comment on video, and positive sentiment scores (Table 5). Sponsorship, commenter's status, overall comment on video, focus of concern, and perceived Invisalign's disadvantages were significantly associated with increased negative sentiment scores (Table 5).

\section{Discussion}

This study shows that YouTube users viewed massively videos related to Invisalign treatment and interacted with the creators and fellow viewers. Completeness of information, video duration, and lifespan did not significantly influence the numbers of views, subscriptions, likes, dislikes, and comments. On this basis, the viewing behavior of YouTube audience may be considered unpredictable, determined by random factors.

Almost half of the vloggers were treated by a dentist, a fact that reflects the reality in provision of orthodontic care in the US, where general dental offices have been

Table 5 values of the Pearson's chi-squared test for sponsorship, commenter's status, and comment classification

\begin{tabular}{lll}
\hline & \multicolumn{2}{l}{$P$ value } \\
\cline { 2 - 3 } & Positive sentiment & Negative sentiment \\
\hline Sponsorship & 0.55 & $<0.001^{*}$ \\
Commenter's status & $<0.001^{*}$ & $<0.001^{*}$ \\
Overall comment & $<0.001^{*}$ & 0.03 \\
Focus of concern & 0.13 & $<0.001^{*}$ \\
Invisalign's disadvantages & 0.27 & $<0.001^{*}$ \\
\hline
\end{tabular}

*Statistically significant at $p<0.05$ carrying out up to $48.9 \%$ of orthodontic procedures [21]. Hypothetically, a similarly high prevalence of dentists may be expected in the 100,000 certified Invisalign providers around the world [9]. Comparison in the use of Invisalign showed that orthodontists treated generally more Invisalign cases, though dentists were building faster their caseload [22]. General dentists appeared also more willing to treat more complicated malocclusions with Invisalign $[22,23]$ adhere less to the digital treatment plan, and use fewer auxiliaries, perhaps demonstrating a difference in treatment goals [23].

Treatment procedures as well as complications were among the main themes identified in Internet discussion forums visited by orthognathic patients [24]. Problems experienced with braces were also primarily posted by orthodontic patients in Twitter communication [25]. IER or refinements were rarely described by the vloggers. Nonetheless, the Invisalign process is not relied on aligners alone. It requires the standard use of auxiliaries like attachments, interarch elastics, IER, and altered aligner geometries to improve the predictability of orthodontic movement [8]. Pain and oral symptoms were most frequently reported by vloggers, while they were also highly viewed as Invisalign's disadvantage by the audience. Notwithstanding, Invisalign tends to cause less pain compared to fixed appliances during the initial stages of treatment, relatively high levels of pain may be anticipated in the first days after insertion [26] or after tray deformation [27]. Several vloggers complained about the need to brush teeth after snacking or tooth sensitivity that made them to adjust the frequency or type of meals ("Invisalign-diet"), which may question the advantage of Invisalign that allows aligner patients "to enjoy all foods" as Align Technology argues [28]. Speech impairment was frequently noted as side effect in the video testimonials. Patients with acrylic plates experienced significantly more pronounced speech difficulties than others with fixed appliances [29] and vacuum-formed retainers [30]. Plenty of viewers appeared to confuse Invisalign aligners with retainers, which might indicate a possible gap in providing sufficient information by specialists during consultation [24]. It is the anonymity of social media that allows patients to communicate topics that they felt uncomfortable to discuss in person or selfperceived inappropriate to ask directly to health care professionals [31].

Narratives are believed to provide essential emotional and social information not usually available through routine resources. Another's experience helps others to understand their medical condition, cope, and adjust to treatment regimens [32]. Commenters were mainly former, active Invisalign patients or eager to start treatment and shared information about their treatment 
experience, occlusion, or even found similarities with the vlogger's description. Like other patients participating in Internet forums [24], Invisalign patients sought online additional information, support, and reassurance from peers undergoing the same process.

A multifaceted audience was actively involved in watching Invisalign testimonials and sharing comments online. Besides channel subscribers or candidates for clear aligners, vloggers, professionals (orthodontists, dentists, practice staff, lab technician), the manufacturer, product promoters (i.e., vibration device claimed to facilitate tooth movement, aligner seating tool), and research recruiters intervened by posting comments. The interest of different viewers' groups confirms the claimed potential of social media to monitor public response to health issues, identify misinformation, and identify targeted areas for intervention efforts [33].

As YouTube users commonly engage in active discussion by expressing either positive or negative emotions in their messages [34], this study further analyzed the emotional content of the comments about the vlogger's experience with Invisalign. Overall, the positive loading of the comments was significantly more pronounced than the negative loading, though both positive and negative mean sentiment scores indicated mild emotions. Likewise, a recent Twitter analysis of the patient experience with braces vs Invisalign revealed more positive tweets about orthodontic treatment without significant differences in sentiment between braces and Invisalign tweets [13]. Viewer's status, comment content, and sponsorship of videos were significantly associated with the sentiments lying behind the comments. Paid patient testimonials are nowadays commonplace across various specialties. Dental, ophthalmologic, and plastic surgery clinics are routinely recruiting patients to help advertise-witness their experience on video testimonials offering treatment discounts [35]. In the present study, the viewers questioned the consumer's opinion in sponsored reviews and expressed negative comments about the reliability and the motives of the vlogger implying bias driven by financial interests.

Narrative communication through social media can improve users' learning abilities by providing ideal health role models. On the contrary, sharing information within personal stories may also hide risks, especially when acting as a deterrent for patients from visiting health professionals or the public may not know how to correctly apply online information about their personal health situation. Since videos involving patient experiences are of lower educational value than expert led ones [36], professional associations and academic departments need to take the lead, to develop and disseminate online evidence-based educational videos. Several YouTube and Vimeo video links (i.e., patient testimonials, instructions about eating, brushing during orthodontic treatment, retention, etc.) are available on the Web site of the American Association of Orthodontists (AAO). Additionally, AAO has created its own YouTube channel, which currently hosts more than 50 videos that basically aim to promote the benefits of orthodontics to the larger society. By expanding topics to standard and innovative techniques and materials, users of video sharing platforms will get access to reliable and updated patient education materials. Clinicians should assist patients in navigating social media and embed on the web pages of their practices links to valid information sources [5].

\section{Study strengths and limitations}

This is the first study to investigate information sharing and interaction trends among YouTube users regarding Invisalign treatment. The use of qualitative research in health care enables an in-depth understanding of patients' thoughts and experiences [24]. Since the researcher is not present during data collection and the conversations analyzed occur naturally, the introduction of bias by the researcher is prevented. Automated sentiment analysis by means of SentiStrength is useful in processing short comments because it extracts both positive and negative sentiments contained in textual statements [37]. Moreover, SentiStrength outperforms other lexical classifiers [36] and therefore may be assumed to have strengthened our methodology.

Like in most studies on consumer health YouTube videos [37], the first five pages of search results were reviewed. A more sophisticated "snowballing" than a sequential screening approach has been recommended instead to identify relevant content through the suggested videos generated by YouTube algorithm [11]. However, it remains still unclear whether such strategy represents the common practice in YouTube searches. Taking into consideration the YouTube dynamics, the search results and the study implications apply only for the specific search date. Albeit the five-point rating scale used in this study was selfdeveloped and not pre-validated, it provided to some degree a measure of the comprehensiveness of information covering treatment aspects that typically concern users of social media and online discussion groups [12, 24, 25]. Future research should further invest in monitoring the orthodontic interests and sentiments of social media users and developing patient education interventions that meet patients' expectations and needs.

\section{Conclusions}

- There is an intense activity on YouTube on obtaining information regarding treatment with Invisalign aligners. 
- The emotional loading of viewers' comments about Invisalign aligners was significantly more positive and was significantly associated with their status, content, and sponsorship of videos.

- Range of information, video duration, and time since upload did not significantly influence engagement and view metrics, a finding that warns against the unpredictable viewing preferences of YouTube users, and the incomplete information in popular Invisalign patient testimonials.

\section{Acknowledgements}

The authors wish to thank Professor M. Thelwall, Faculty of Science and Engineering, University of Wolverhampton, UK, for kindly granting access to SentiStrength software for the purposes of the study.

\section{Authors' contributions}

$\mathrm{CL}$ and $\mathrm{KD}$ designed the study, collected and interpreted data, and drafted paper. NP analyzed and interpreted data. All authors read and approved the final manuscript.

\section{Competing interests}

The authors declare that they have no competing interests.

\section{Publisher's Note}

Springer Nature remains neutral with regard to jurisdictional claims in published maps and institutional affiliations.

\section{Author details}

'Department of Orthodontics, Academic Centre for Dentistry Amsterdam (ACTA), University of Amsterdam and VU University Amsterdam, Amsterdam, The Netherlands. ${ }^{2}$ Department of Oral and Maxillofacial Surgery, University of Groningen, University Medical Center Groningen, Groningen, The Netherlands. ${ }^{3}$ Department of Orthodontics and Dentofacial Orthopaedics, School of Dental Medicine, University of Bern, Bern, Switzerland. ${ }^{4}$ Private practice, Corfu, Greece.

Received: 6 November 2017 Accepted: 17 December 2017

Published online: 22 January 2018

\section{References}

1. Fagerlin A, Wang C, Ubel PA. Reducing the influence of anecdotal reasoning on people's health care decisions: is a picture worth a thousand statistics? Med Decis Mak. 2005;25:398-405.

2. Huh J, Liu LS, Neogi T, Inkpen K, Pratt W. Health vlogs as social support for chronic illness management. ACM Trans Comput Hum Interact. 2014:21:23.

3. Alexa. youtube.com Traffic Statistics. http://www.alexa.com/siteinfo/youtube. com. Accessed 7 Aug 2017.

4. Knösel M, Jung K. Informational value and bias of videos related to orthodontics screened on a video-sharing Web site. Angle Orthod. 2011; 81(3):532-9.

5. Delli K, Livas C, Vissink A, Spijkervet FK. Is YouTube useful as a source of information for Sjögren's syndrome? Oral Dis. 2016;22:196-201.

6. Al-Silwadi FM, Gill DS, Petrie A, Cunningham SJ. Effect of social media in improving knowledge among patients having fixed appliance orthodontic treatment: a single-center randomized controlled trial. Am J Orthod Dentofac Orthop. 2015;148:231-7.

7. Keim RG, Gottlieb E, Vogels DS 3rd, Vogels PB. JCO study of orthodontic diagnosis and treatment procedures, Part 1: results and trends. J Clin Orthod. 2014:48:607-30.

8. Rossini G, Parrini S, Castroflorio T, Deregibus A, Debernardi CL. Efficacy of clear aligners in controlling orthodontic tooth movement: a systematic review. Angle Orthod. 2015;85:881-9.

9. Invisalign. 4.5 Million Smiles. http://invisaligncenter.ae/en/blog/4-5-millionsmiles. Accessed 14 Aug 2017.

10. Fat MJL, Sell E, Barrowman N, Doja A. Public perception of tourette syndrome on YouTube. J Child Neurol. 2012;27(8):1011-6.
11. Sampson M, Cumber J, Li C, Pound CM, Fuller A, Harrison D. A systematic review of methods for studying consumer health YouTube videos, with implications for systematic reviews. PeerJ. 2013;1:e14.

12. Detels R, Gulliford M, Karim QA, Tan CC. New communication technologies, social media, and public health, Oxford textbook of global public health, vol. 1. 6th ed. Oxford: Oxford University Press; 2015. p. 391.

13. Noll D, Mahon B, Shroff B, Carrico C, Lindauer SJ. Twitter analysis of the orthodontic patient experience with braces vs Invisalign. Angle Orthod. 2017:87:377-83.

14. Pang B, Lee L. Opinion mining and sentiment analysis. Found Trends Inf Retr. 2008:2:1-135

15. Suvakov M, Mitrovic M, Gligorijevic V, Tadic B. How the online social networks are used: dialogues-based structure of MySpace. J R Soc Interface. 2013;10:20120819

16. Iosub D, Laniado D, Castillo C, Fuster Morell M, Kaltenbrunner A. Emotions under discussion: gender, status and communication in online collaboration. PLoS One. 2014:9:e104880.

17. Bhattacharya S, Srinivasan P, Polgreen P. Engagement with health agencies on twitter. PLoS One. 2014:9:e112235.

18. Rodrigues RG, das Dores RM, Camilo-Junior CG, Rosa TC. SentiHealth-Cancer: a sentiment analysis tool to help detecting mood of patients in online social networks. Int J Med Inform. 2016;85:80-95.

19. Charlton N, Singleton C, Greetham DV. In the mood: the dynamics of collective sentiments on Twitter. R Soc Open Sci. 2016;3:160162.

20. Thelwall M, Buckley K, Paltoglou G, Cai D, Kappas A. Sentiment strength detection in short informal text. J Am Soc Inf Sci. 2010;61:2544-58.

21. del Aguila MA, Anderson M, Porterfield D, Robertson PB. Patterns of oral care in a Washington State dental service population. J Am Dent Assoc. 2002;133:343-51.

22. Vicéns J, Russo A. Comparative use of Invisalign by orthodontists and general practitioners. Angle Orthod. 2010;80:425-34

23. Best AD, Shroff B, Carrico CK, Lindauer SJ. Treatment management between orthodontists and general practitioners performing clear aligner therapy. Angle Orthod. 2017:87:432-9.

24. Bhamrah G, Ahmad S, NiMhurchadha S. Internet discussion forums, an information and support resource for orthognathic patients. Am J Orthod Dentofac Orthop. 2015;147:89-96

25. Rachel Henzell M, Margaret Knight A, Morgaine KC, Antoun JS, Farella M. A qualitative analysis of orthodontic-related posts on Twitter. Angle Orthod. 2014:84:203-7.

26. Shalish M, Cooper-Kazaz R, Ivgi I, Canetti L, Tsur B, Bachar E, Chaushu S. Adult patients' adjustability to orthodontic appliances. Part l: a comparison between Labial, Lingual, and Invisalign ${ }^{T M}$. Eur J Orthod. 2012;34:724-30.

27. Fujiyama K, Honjo T, Suzuki M, Matsuoka S, Deguchi T. Analysis of pain level in cases treated with Invisalign aligner: comparison with fixed edgewise appliance therapy. Prog Orthod. 2014;15:64.

28. Invisalign. The Invisalign ${ }^{\oplus}$ Advantage. https://www.invisalign.com/whyinvisalign/invisalign-advantage. Accessed 14 Aug 2017

29. Wiedel AP, Bondemark L. A randomized controlled trial of self-perceived pain, discomfort, and impairment of jaw function in children undergoing orthodontic treatment with fixed or removable appliances. Angle Orthod. 2016:86:324-30

30. Saleh M, Hajeer MY, Muessig D. Acceptability comparison between Hawley retainers and vacuum-formed retainers in orthodontic adult patients: a single-centre, randomized controlled trial. Eur J Orthod. 2017:39:453-61

31. Taggart T, Grewe ME, Conserve DF, Gliwa C, Roman Isler M. Social media and HIV: a systematic review of uses of social media in HIV communication. Med Internet. 2015;17:e248.

32. Bekker HL, Winterbottom AE, Butow $P$, Dillard AJ, Feldman-Stewart $D$, Fowler FJ, Jibaja-Weiss ML, Shaffer VA, Volk RJ. Do personal stories make patient decision aids more effective? A critical review of theory and evidence. BMC Med Inform Decis Mak. 2013;13(Suppl 2):S9.

33. Moorhead SA, Hazlett DE, Harrison L, Carroll JK, Irwin A, Hoving C. A new dimension of health care: systematic review of the uses, benefits, and limitations of social media for health communication. J Med Internet Res. 2013:15:e85.

34. Oksanen A, Garcia D, Sirola A, Näsi M, Kaakinen M, Keipi T, Räsänen P. Pro-anorexia and anti-pro-anorexia videos on YouTube: sentiment analysis of user responses. J Med Internet Res. 2015;17:e256.

35. Ellin A. Coming soon to YouTube: My facelift. http://cg-ins.com/comingsoon-to-youtube-my-face-lift. Accessed 10 Aug 2017. 
36. Strychowsky JE, Nayan S, Farrokhyar F, MacLean J. YouTube: a good source of information on pediatric tonsillectomy? Int J Pediatr Otorhinolaryngol. 2013;77:972-5

37. Nielsen FÅ. A new ANEW: Evaluation of a word list for sentiment analysis in microblogs. https://arxiv.org/abs/1103.2903. Accessed 14 Aug 2017.

\section{Submit your manuscript to a SpringerOpen ${ }^{\bullet}$} journal and benefit from:

- Convenient online submission

- Rigorous peer review

- Open access: articles freely available online

- High visibility within the field

- Retaining the copyright to your article

Submit your next manuscript at $\gg$ springeropen.com 